\title{
A Probe on Localization Techniques in Wireless Sensor Networks
}

\author{
Martin Victor $\mathrm{K}$ \\ PG Scholar, Department of CSE, \\ School of Computer Science \& Technology, \\ Karunya University, \\ Karunya Nagar, Coimbatore - 641114. \\ Tamilnadu, INDIA.
}

\author{
K. Ramalakshmi \\ Assistant Professor (SG), Department of CSE, \\ School of Computer Science \& Technology, \\ Karunya University, \\ Karunya Nagar, Coimbatore - 641114 \\ Tamilnadu, INDIA.
}

\begin{abstract}
Coverage in wireless sensor networks is typically defined as a determination of how correctly and for how long the sensors are proficient to monitor the physical space. It can be taken as the consideration for evaluating the quality of service. Since sensors are spread in a random manner, one of the primary issues in wireless sensor networks is the coverage problem. It is an issue which is to be determined whether a region is sufficiently covered by a set of sensors. The deployment and activation of static nodes along with the density control and trajectory of mobile nodes are major concerns of the coverage problem and the same should be dealt upon through competent localization techniques. This work analyzes localization based on the hardware, design and techniques under several classifications and finally outlines the current state of contemporary localization techniques accompanied with the inherent design tradeoffs.
\end{abstract}

\section{Keywords}

Wireless Sensor Networks; Localization; Range-based; Range-free.

\section{INTRODUCTION}

A wireless sensor network (WSN) consists of spatially distributed independent sensors to observe physical or environmental conditions, such as temperature, sound, pressure, etc. and to considerately forward their data through the network. The WSN is formed of nodes - from a very little to many, where every node is linked to one or numerous sensors. In the field of Wireless Sensor Networks Technology, Localization plays an important role. Here, Localization problem means the method of approximating and figuring out the positions of sensor nodes. The significance of this information directed the researchers to find a solution for it. One way is the manual design configuration but this is very unworkable in wide-ranging or when sensors are deployed in remote areas or when sensors are movable. Another way is adding the global positioning system (GPS) to each sensor. Since GPS requires line-of-sight between the receiver and satellites, it has exaggerated heavy trees and big buildings. Due to poor signal reception it has low accuracy.

\section{LOCALIZATION METHOD}

As it is mentioned earlier, localization means finding the position of the individual sensor nodes. In general, the localization method can be classified into four different categories:
- Centralized vs Distributed

- Anchor-free vs Anchor-based

- Range-free vs Range-based

- Mobile vs Stationary

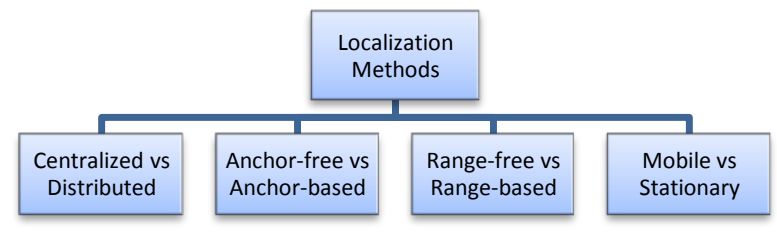

Fig 1 - Classification of Localization Method

But here, the survey is focused only on Range-free vs Rangebased and so, according to this position, Localization can be grossly divided into two categories:

- Range-based Localization

- Range-free Localization

\subsection{Range-based Localization}

The Range-based Localization is described by set of rules that apply absolute point-to-point distance ballpark figure (range) or angle estimates for evaluating the locations. For Rangebased Localization, there are four processes:

- $\quad$ RSSI-based Algorithms

- RSSI do not require Static Beacons

- Precise ranging technologies

- Camera-based ranging scheme

\subsubsection{RSSI-based Algorithms}

RSSI is used by RADAR [1] for constructing a centralized storage area of signal strengths which is at different positions with respect to a set of beacons. RADAR, a radio frequency (RF) based system which is for positioning and trailing users inside the buildings is presented here. The operation is done by recording and processing the signal strength information at numerous base stations located to offer coinciding coverage in the area of interest. For determining the user location, RADAR combines the experimental measurements with the signal propagation modeling and thereby, location-aware 
services and applications are enabled. RADAR completely stands on the experimental signal strength measurements as well as an easy yet strongly operative signal propagation model.

The merits of RADAR are the signal propagation method makes deployment easier, the positioning and trailing users with a high degree of accuracy is possible. The demerit is to mingle position information examines with the RADAR system and to set up this within an institute is not available.

Motetrack [2] circulates the values of suggested RSSI to beacons. Robust which is a decentralized methodology to RFbased location tracking is used here. Motetrack is based on stumpy power radio transceivers tied with a meek amount of calculation and storage abilities. It is independent on any back-end server or network infrastructure. The position of each moving node is evaluated utilizing a received radio signal strength signature from various beacon nodes to a folder of signatures that is imitated across the beacon nodes themselves. This pattern permits the system to process in spite of noteworthy failures of the radio beacon infrastructure. Here, a 50th percentile and 80th percentile position-tracing correctness of 2 meters and 3 meters respectively is achieved in the installation of Motetrack which comprises of 20 beacon nodes scattered through a Computer Science building. Additionally, Motetrack can accept the collapse of up to $60 \%$ of the beacon nodes exclusive of severely humiliating accuracy by making the system appropriate for deployment in highly unpredictable conditions. The merit of Motetrack is, it can attain up to 50th and 80th percentile of 2 and 3 meters respectively.

\subsubsection{RSSI not requiring Static Beacons}

Networked Robots [3] is the application of a sensor network to steer a flying robot. The disseminated algorithms and wellorganized geographic routing techniques are developed to incrementally direct one or more robots to positions of interest which is based on sensor inclined fields, or described in conditions of Cartesian coordinates. The robot itself is the primary component of the localization process that launches the locations of sensors which are unknown a priori. This process is used in a wide-range outdoor experiment with Mote sensors to lead a self-directed helicopter along a path fixed in the network. The merit of Networked robots is the helpfulness of geographic or vector routing and the demerit is the assembling of data from robot steering trials.

A localization scheme based on a single mobile beacon [6] which is attentive of its position is presented here. Sensor nodes getting the beacon sachets deduce propinquity limitations to the moving beacon and use them to build and uphold location estimates. This system is RF-based and thus no additional hardware is needed. The correctness is adequate for most applications. The accomplishment is used to assess the functioning of this approach. A localization process is anticipated using Bayesian conjecture for dealing out the information from single mobile beacon. A beacon is a node attentive of its position. The unknown nodes are the nodes of which its initial positions are not known. Once the sensor node is arranged, the mobile beacon helps the nodes which are not known in localizing themselves. The moving beacon can be a human machinist, an unmanned vehicle arranged with the sensor network, or even a plane when in the case of a deployment from plane. The merit here is, it provides an unpredictably excellent correctness.

\subsubsection{Precise Ranging Technologies}

Cricket [7] is a locality bearing system for in-building, itinerant, position dependent functions. It permits applications operating on dynamic and static nodes to study their substantial location by utilizing the spectators that hear and examine the information from beacons proliferated all over the building. Cricket is the outcome of a number of design goals together with user seclusion, decentralized administration, complex heterogeneity and squat price. The haphazard algorithm is illustrated here which is used by beacons to broadcast the information, the exploitation of simultaneous radio and ultrasonic signals to deduce distance, the listener deduction algorithms to triumph over multipath and intrusion, and realistic beacon pattern and localizing procedures that increase accuracy. The merits of Cricket are user privacy, network heterogeneity and portion of a room granularity.

\subsubsection{Camera-based ranging scheme}

A Camera-based ranging scheme is presented where two or more cameras work together to identify the nodes in their communal provinces of analysis. Camera Calibration [10] in distributed camera sensor networks observes node localization and camera calibration utilizing the mutual province of view of camera pairs. By using a new distributed camera sensor network, there are two tactics from computer hallucination are compared and an algorithms that merges a meager set of distance dimensions with picture information to precisely locate the nodes in 3D is anticipated. These algorithms are assessed using a network of iMote 2 nodes prepared with COTS camera modules. The sensor nodes detect themselves to camera by using adapted LED emanations. The merit of Camera Calibration is, it permits to control the association of imager and non-imager sensors to detect other trials, goals and activities in sensor networks. The demerit is using of reconfigurable middleware structure to locate and trace events other than sensor nodes is not available.

\subsection{Range-free Localization}

Range-free approaches present a substitute to the trials and costs forced by range-based schemes. These approaches locate the nodes by controlling simply detectable, countable occurrence in the situations together with the hop counts to neighbors and the entry and exit of the emitters in the area. Under this, there are three categories:

- Centtroid Algorithm

- DV-HOP

- Amorphous Positioning 


\subsubsection{Centroid Algorithm}

Range-Free Localization Schemes for Wide range Sensor Networks [13] suggests APIT, where nodes choose the location based on the prospect of being inside a triangle formed by three beacons. APIT is a novel localization algorithm which is a range-free one is presented here to perform. APIT scheme achieves well when an asymmetrical radio model and arbitrary node arrangement are deliberated, and stumpy interaction overhead is preferred. This effort is related through widespread replication, with three positions of the art range-free schemes to detect the desirable process. Additionally, the study on the consequence of position faults on routing and tracing performance is also done. The merit here is, it achieves well when an asymmetrical radio model and arbitrary node arrangement are deliberated and stumpy interaction overhead is preferred. The demerit here is, it displays that the accuracy delivered by the range-free schemes pondered in sensor networks with only trivial enactment of deprivation.

\subsubsection{DV-HOP}

Global Coordinate System [14] practices a priori information of node concentration to evaluate typical hop distance. It is feasible to attain precise localization and tracing of a goal in a randomly situated wireless sensor networks collected of reasonable mechanisms of restrained accuracy. The vital enabler for this is a sensibly correct local coordinate system affiliated with the large-scale coordinates. An algorithm is offered here for generating such a coordinate system exclusive of the use of wider control, globally manageable beacon signals, or exact approximation of inter sensor distances. The coordinate system is hardy and robotically adjusts to the disaster or addition of sensors. Widespread hypothetical analysis and imitation outcomes are presented. The merit here is the ability to attain very realistic accuracy and the failure is hypothetically analyzable. The demerit is, an enactment of tracing a drifter in an arena tenanted by sensors is not available.

\subsubsection{Amorphous Positioning}

Sensor node Localization Using Uncontrolled Events [17] is a concrete design utilizing completely uncontrolled events for immobile sensor node localization. The novel impression of this design is to evaluate both the event generation boundaries and the positioning of each sensor node by handling node arrangements simply attained from uncontrolled event dissemination. To determine the overview of the design, both straight-line scan and circular wave proliferation events are focused here, and this technique is calculated through hypothetical exploration, broad simulation and a physical testbed execution with $41 \mathrm{MICAz}$ motes. Additionally, localization utilizing uncontrolled events offers a good prospective preference of attaining node localizing through natural ambient events. The merit here is the sensor node localization is attainable with great tractability, low cost and good accuracy. The demerit here is the unavailability of localizing the sensor nodes using controlled events.

\section{EVENT-BASED LOCALIZATION}

A great precision, Low-Cost Locating System for Wireless Sensor Networks [18] is a scheme which properly defines, enterprises, performs and assesses a novel localization system, called Spotlight. Here, the spatio-temporal properties of well controlled events in the network are used to attain the positions of the sensor nodes. A high correctness in localization is established which can be attained without the help of high cost hardware on the sensor nodes, as needed by the other localization systems. The demerit here is the study of self-correction and self-tuning of the Spotlight system is not available.

\section{COMPARISON}

Here, all the protocols are compared with respect of the methods used in the respective papers and also the merits and demerits of each protocol. Based on the comparison, the basic idea of each paper can be known and through that, the overall survey on these protocols is done. Table 1 refers the comparison between the different protocols.

Table 1 - Comparison between the different protocols

\begin{tabular}{|c|c|c|c|}
\hline Protocol & Techniques & Merits & Demerits \\
\hline RADAR [1] & $\begin{array}{c}\text { Radio Frequency (RF) based } \\
\text { System }\end{array}$ & $\begin{array}{c}\text { Norm resolution is in the } \\
\text { range of } 2 \text { to } 3 \text { meters, about } \\
\text { the size of a typical office } \\
\text { room }\end{array}$ & $\begin{array}{c}\text { Deployment is unavailable } \\
\text { within an organization }\end{array}$ \\
\hline MoteTrack [2] & Low power radio transceivers & $\begin{array}{c}\text { Achievement of percentiles of } \\
50^{\text {th }} \text { and } 80^{\text {th }} \text { of } 2 \text { and 3 } \\
\text { meters respectively }\end{array}$ & Time consuming \\
\hline Networked Robots [3] & $\begin{array}{c}\text { Application of a sensor } \\
\text { network to navigate a flying } \\
\text { robot }\end{array}$ & $\begin{array}{c}\text { Effectiveness of geographic } \\
\text { or vector routing }\end{array}$ & $\begin{array}{c}\text { Demonstrating sensor-based } \\
\text { path adaptation }\end{array}$ \\
\hline Mobile Beacon [6] & $\begin{array}{c}\text { To construct and maintain } \\
\text { position estimates }\end{array}$ & $\begin{array}{c}\text { Unexpectedly good accuracy } \\
\text { about a sort of scale better } \\
\text { than existing methods }\end{array}$ & Less security \\
\hline Cricket [7] & $\begin{array}{c}\text { In-building, mobile, location } \\
\text { dependent applications }\end{array}$ & User privacy & Unavailability of \\
\hline Camera-Based [10] & Using the shared field of view & Identification of other events, & Locy \\
\hline
\end{tabular}




\begin{tabular}{|c|c|c|c|}
\hline & of camera pairs & $\begin{array}{c}\text { targets and behaviors in } \\
\text { sensor net }\end{array}$ & $\begin{array}{c}\text { reconfigurable middleware } \\
\text { framework }\end{array}$ \\
\hline Range-free [13] & $\begin{array}{c}\text { APIT (Approximate Point In } \\
\text { Triangulation) }\end{array}$ & $\begin{array}{c}\text { Good performance when } \\
\text { asymmetrical radio patterns } \\
\text { are deliberated }\end{array}$ & $\begin{array}{c}\text { Only slight performance } \\
\text { degradation }\end{array}$ \\
\hline Ad Hoc [14] & $\begin{array}{c}\text { Estimating average hop } \\
\text { distance }\end{array}$ & $\begin{array}{c}\text { Acclimatizes to procure gain } \\
\text { of better sensor potentials }\end{array}$ & $\begin{array}{c}\text { Unavailability of tracking } \\
\text { rover in a field }\end{array}$ \\
\hline Uncontrolled Events [17] & $\begin{array}{c}\text { Using uncontrolled events for } \\
\text { stationary sensor node } \\
\text { positioning }\end{array}$ & $\begin{array}{c}\text { Great flexibility and good } \\
\text { accuracy with uncontrolled } \\
\text { events }\end{array}$ & $\begin{array}{c}\text { Unavailability of controlled } \\
\text { events }\end{array}$ \\
\hline Event-Based [18] & $\begin{array}{c}\text { Spotlight uses properties of } \\
\text { well controlled events }\end{array}$ & $\begin{array}{c}\text { Locating the position of the } \\
\text { node with controlled events }\end{array}$ & $\begin{array}{c}\text { Unavailability of the study of } \\
\text { self-correction and self- } \\
\text { tuning of the Spotlight system }\end{array}$ \\
\hline
\end{tabular}

\section{CONCLUSION}

This paper describes about the survey made in the prior work on the localization techniques. The major classification of the localization method is articulated and its respective techniques are discussed. The localization based on the hardware, design and techniques under several classifications are analyzed.

\section{REFERENCES}

[1] "Radar: An in-Building Rf-Based User Location and Tracking System," Proc. IEEE INFOCOM, 2000 Bahl and Padmanabhan.

[2] K. Lorincz and Welsh, "Motetrack: A Robust Decentralized Approach to RF-Based Location Tracking," Proc. Int'l Workshop Location and ContextAwareness, 2005.

[3] Corke. P, Peterson. R, and Rus. D, "Networked Robots: Flying Robot Navigation Using a Sensor Net," Proc. Int'l Symp. Robotics Research (ISRR), 2003.

[4] "Localization for Mobile Sensor Networks," Proc. ACM MobiCom, 2004 L. Hu and D. Evans.

[5] Pathirana, Savkin, Jha, and Bulusu, "Node Localization Using Mobile Robots in Delay-Tolerant Sensor Networks," IEEE Trans. Mobile Computing, vol. 4, no. 3, pp. 285-296, May/June 2005.

[6] "Localization of Wireless Sensor Networks with a Mobile Beacon," Proc. IEEE Int'l Conf. Mobile Ad-Hoc and Sensor Systems, 2004 M. Sichitiu and V. Ramadurai.

[7] Priyantha, Chakraborty, and Balakrishnan, "The Cricket Location-Support System," Proc. ACM MobiCom, 2000.

[8] "Dynamic Fine-Grained Localization in Ad-Hoc Networks of Sensors," Proc. ACM MobiCom, 2001 A.Savvides, C. Han, and M. Srivastava.
[9] "Sensor Network-Based Countersniper System," 2004 , Simon, Maroti, Ledeczi, Balogh, Kusy, Nadas, Pap, Sallai, and Frampton.

[10] Barton-Sweeney, Lymberopoulos, and Savvides, "Sensor Localization and Camera Calibration in Distributed Camera Sensor Networks," Proc. Third Int'l Conf. Broadband Comm.,Networks and Systems (BROADNETS), pp. 1-10, 2006.

[11] "GPS-Less Low Cost Outdoor Localization for Very Small Devices," IEEE Personal Comm. Magazine, vol. 7, no. 5, pp. 28-34, Oct. 2000, N. Bulusu, J. Heidemann, and D. Estrin.

[12] Schuhmann, Herrmann, Rothermel, Blumenthal, and D.Timmermann, "Improved Weighted Centroid Localization in Smart Ubiquitous Environments," 2008.

[13] He, Huang, Blum, Stankovic, and Abdelzaher, "RangeFree Localization Schemes in Large Scale Sensor Networks,” Proc. ACM MobiCom, 2003.

[14] "Organizing a Global Coordinate System from Local Information on an Ad Hoc Sensor Network," Proc.Second Int'l Conf. Information Processing in Sensor Networks (IPSN), 2003, R. Nagpal, H. Shrobe, and J. Bachrach.

[15] Niculescu and B. Nath, "Ad-Hoc Positioning System," Proc. IEEE Global Telecomm. Conf. (GLOBECOM), 2001.

[16] "MSP: Multi-Sequence Positioning of Wireless Sensor Nodes," Proc. Int'l Conf. Embedded Networked Sensor Systems (SenSys), 2007, Zhong and T. He.

[17] Zhong, D. Wang, and He, "Sensor Node Localization Using Uncontrolled Events," Proc. Int'l Conf. Distributed Computing Systems (ICDCS), 2008.

[18] "A High-Accuracy Low-Cost Localization System for Wireless Sensor Networks," Proc. Int'l Conf. Embedded Networked Sensor Systems (ENSS), 2005, R. Stoleru, T. He, J.A. Stankovic, and D. Luebke. 